\title{
Challenging the convention of utilizing actual body weight dosing with intravenous immune globulin
}

\author{
Elizabeth Wojakowski ${ }^{1}$, Brian Kersten ${ }^{2}$ and Ashley E Woodruff ${ }^{*}$ \\ ${ }^{1}$ University at Rochester Medical Center, Rochester, NY 14642, USA \\ ${ }^{2}$ Buffalo General Medical Center, Buffalo, NY 14203, USA \\ ${ }^{3}$ Pharmacy Practice and Internal Medicine, School of Pharmacy and Pharmaceutical Sciences and Jacobs School of Medicine and Biomedical Sciences, University \\ at Buffalo, USA
}

Intravenous immune globulin (IVIG) is indicated for the treatment of primary immunodeficiency, certain autoimmune/inflammatory conditions and prevention of bacterial infections in hematologic malignancies. It is traditionally dosed using actual body weight (ABW) [1], however, an expansive list of off-label IVIG uses has generated increased attention on the economic impact of this conventional dosing strategy. An assessment of the pharmacokinetic (PK) profile of IVIG suggests the practice of ABW based dosing could be called into question. IVIG has a small volume of distribution and lacks accumulation in lipophilic tissues [2]. Medications with similar PK profiles, including acyclovir are dosed using ideal body weight (IBW) [3]. Utilizing IBW dosing has been shown to conserve usage and reduce cost [4], but there is limited data regarding the efficacy of this dosing strategy. Traditionally, IVIG doses are based on body weight and given for a discrete time period or titrated to clinical effect. There is literature, though limited, to suggest an association between the magnitude of change in serum IgG levels pre-post IVIG therapy and clinical response $[5,6]$. To evaluate various IVIG dosing strategies and their effect on changes in serum IgG levels pre-post IVIG therapy, Anderson and Olson retrospectively evaluated eleven adult and seven adolescent patients to compare IgG levels before and after therapy with IVIG dosing based on ABW, IBW, and adjusted body weight. The correlation between IgG levels pre-post IVIG therapy was strongest, though not statistically significant, with dosage based on IBW ( $\mathrm{r}=0.83$ vs 0.73 , $\mathrm{p}=0.65$ for IBW vs ABW dosing in adult patients) [7]. In patients with hematologic malignancies, IVIG dosage strategies involving ABW, IBW or adjusted body weight (adjBW) were compared in 209 patients. There was no difference in infection rate between patients treated with an ABW strategy vs those treated with an IBW or adjBW strategy and IgG-level response after IVIG administration was not different between dosing groups. The authors suggested that use of IBW/adjBW dosing strategies could provide significant monthly savings compared to IVIG cost when using ABW dosing [8]. Other authors have experimented with smaller doses titrated to observed clinical response, reflecting a trend toward precision medicine, however this has not been studied in all populations and is not practical for all indications [9,10]. Currently, there is no data directly evaluating clinical outcomes using IBW versus ABW based IVIG regimens to guide the optimal dosing strategy in autoimmune/inflammatory conditions. At current, dosing IVIG based

Copyright: (C2018 Wojakowski E. This is an open-access article distributed under the terms of the Creative Commons Attribution License, which permits unrestricted use, distribution, and reproduction in any medium, provided the original author and source are credited. on IBW provides an economically attractive, alternative approach to conventional ABW dosing, however additional investigation is needed regarding clinical efficacy.

\section{Conflicts of interest}

All authors have nothing to disclose and no conflicts of interest

\section{References}

1. Ballow M (2005) Clinical and investigational considerations for the use of IGIV therapy. Am J Health Syst Pharm 62: S12-18. [Crossref]

2. Kerr J, Quinti I, Eibl M (2014) Is dosing of therapeutic immunoglobulins optimal? A review of a three-decade long debate in Europe. Front Immunol 5: 1-19.

3. Turner RB, Cumpston A, Sweet M (2016) Prospective, controlled study of acyclovir pharmacokinetics in obese patients. Antimicrob Agents Chemother 60: 1830-1833.

4. Rocchio MA, Hussey AP, Southard RA, Szumita PM (2013) Impact of ideal body weight dosing for all inpatient i.v. immune globulin indications. Am J Health Syst Pharm 70: 751-752. [Crossref]

5. Kuitwaard K, de Gelder J, Tio-Gillen AP, Hop WC, van Gelder T, et al. (2009) Pharmacokinetics of intravenous immunoglobulin and outcome in Guillain-Barre syndrome. Ann Neurol 66: 597-603. [Crossref]

6. Kuitwaard K, de Gelder J, Tio-Gillen AP, Hop WC, van Gelder T, et al. (2009) Pharmacokinetics of intravenous immunoglobulin and outcome in Guillain-Barré syndrome. Ann Neurol 66: 597-603. [Crossref]

7. Anderson CR, Olson JA (2015) Correlation of weight-based i.v. immune globulin doses with changes in serum immunoglobulin G levels. Am J Health Syst Pharm 72: 285-289. [Crossref]

8. Stump SE, Schepers AJ1, Jones AR, Alexander MD, Auten JJ (2017) Comparison of Weight-Based Dosing Strategies for Intravenous Immunoglobulin in Patients with Hematologic Malignancies. Pharmacotherapy 37: 1530-1536. [Crossref]

9. Godeau B, Lesage S, Divine M, Wirquin V, Farcet JP, et al. (1993) Treatment of adult chronic autoimmune thrombocytopenic purpura with repeated high-dose intravenous immunoglobulin. Blood 82: 1415-1421. [Crossref]

10. Godeau B, Caulier MT, Decuypere L (1999) Intravenous immunoglobulin for adult with autoimmune thrombocytopenic purpura: results of a randomized trial comparing 0.5 and $1 \mathrm{~g} / \mathrm{kg}$ b.w. Br J Haematol 107: 716-719.

${ }^{\star}$ Correspondence to: Ashley E Woodruff, Clinical Assistant Professor of Pharmacy Practice and Internal Medicine, University at Buffalo, School of Pharmacy and Pharmaceutical Sciences and Jacobs School of Medicine and Biomedical Sciences, 205 Kapoor Hall, Buffalo, NY 14214, Tel: 716-645-1734; Fax: 716-859-4555; Email: aew7@buffalo.edu

Key words: IVIG, ideal body weight, pharmacokinetic, dosing

Received: July 19, 2018; Accepted: July 27, 2018; Published: July 30, 2018 